KUCP-55

September 25, 2018

\title{
Comments on Closed Bianchi Models
}

\author{
Yoshinisa FUJIWARA, Hideki ISHIHARA, Hideo KODAMA \\ Department of Fundamental Sciences, FIHS, \\ Kyoto University, Kyoto, Japan
}

\begin{abstract}
We show several kinematical properties that are intrinsic to the Bianchi models with compact spatial sections. Especially, with spacelike hypersurfaces being closed, (A) no anisotropic expansion is allowed for Bianchi type V and VII $(A \neq 0)$, and (B) type IV and VI $(A \neq 0,1)$ does not exist. In order to show them, we put into geometric terms what is meant by spatial homogeneity and employ a mathematical result on 3-manifolds. We make clear the relation between the Bianchi type symmetry of space-time and spatial compactness, some part of which seem to be unnoticed in the literature. Especially, it is shown under what conditions class B Bianchi models do not possess compact spatial sections. Finally we briefly describe how this study is useful in investigating global dynamics in (3+1)-dimensional gravity.
\end{abstract}


The so-called Bianchi model in general relativity has been widely studied by many people (see [1][2] for example). In the model, a space-time is assumed to have symmetry, namely spatially homogeneity. Since the gravitational degrees of freedom are reduced to be finite in this model, it gives an appropriate system for us to understand the full complexity of the Einstein equations and physical cosmology described by it. It also serves as a natural background for perturbations and for a study of quantum fields on it. Moreover, this minisuperspace can be used as a toy model for understanding quantum cosmology. In that context one usually assumes that the spatially homogeneous hypersurface is compact without boundary, i.e. closed.

In this paper, we study such a closed Bianchi model from geometrical viewpoint. We show some interesting kinematical properties intrinsic to the closed Bianchi model which follow from the spatial homogeneity and spatial compactness. In order to show them, we utilize a recent mathematical result on threedimensional manifold by Thurston and others. Some of our results were mentioned in some works by other people, but under somewhat restrictive conditions. We do not assume such restrictions in our geometrical argument and shall make clear some points unnoticed in the literature. In the end of our paper, we describe how such study of closed Bianchi models is useful in order to investigate the global dynamical degrees of freedom, which are often called "moduli", of a closed 3-space in $(3+1)$-dimensional gravity.

Let us begin with a general description of homogeneity in geometry. A Riemannian manifold $(M, g)$ is defined to be locally homogeneous, if for every pair of points $x, y \in M$ there are neighborhoods $U$ and $V$ of $x$ and $y$, for which there exists a local isometry mapping $(U, x)$ to $(V, y)$. Such local isometries do not, in general, extend to isometries of the whole $(M, g)$. Let us denote the full group of isometries of $(M, g)$ by $\operatorname{Isom}(M, g)$. Then if for every pair of points $x, y \in M$ there is an isometry $\Phi \in \operatorname{Isom}(M, g)$ such that $\Phi(x)=y$, that is, $\operatorname{Isom}(M, g)$ acts transitively on $M$, one says that $(M, g)$ is (globally) homogeneous. Since it is a standard fact that a simply-connected and locally homogeneous manifold is 
globally homogeneous, the universal covering space $(\widetilde{M}, \widetilde{g})$ of a locally homogeneous manifold $(M, g)$ must be homogeneous. For our convenience, we shall say that a homogeneous manifold $(M, g)$ is simply homogeneous if $\operatorname{Isom}(M, g)$ has a three-dimensional subgroup $G_{3}$ that acts simply-transitively on $M$. In that case, we call the $G_{3}$ a homogeneity group. If the universal covering space of a locally homogeneous manifold is simply homogeneous with a homogeneity group $G_{3}$, then we shall call it a locally homogeneous manifold with a homogeneity group $G_{3}$.

The usual Bianchi model starts with the following assumptions for spatial homogeneity. A three-dimensional Lie group $G_{3}$ acts on a space-time as a group of isometries of the space-time, such that each orbit is a spacelike hypersurface $\Sigma$ on which $G_{3}$ acts simply-transitively. The space-time considered is topologically a product space $\Sigma \times \mathbf{R}$. We have a family of 3-manifolds $(\Sigma, g(t))$ for each $t \in \mathbf{R}$, where $g$ is the spatial metric intrinsic to $\Sigma$. Then it follows that $G_{3}$ acts simplytransitively on each $(\Sigma, g(t))$ as a group of isometries of $(\Sigma, g(t))$. In the above terms, $(\Sigma, g(t))$ is simply homogeneous.

Such a Riemannian manifold is well understood in the context of Bianchi cosmology. (See [1][2] for references and complete information on the definitions and the notations below.) Denote by $C_{I J}^{K}(I, J, K=1 \sim 3)$ the structure constant of the Lie algebra of $G_{3}$ with respect to a certain basis $\left\{\xi_{I}\right\}:\left[\xi_{I}, \xi_{J}\right]=C_{I J}^{K} \xi_{K}$. Then there globally exists on $M$ an invariant basis $\left\{X_{I}\right\}$ which one can choose so that

$$
\left[X_{I}, X_{J}\right]=-C_{I J}^{K} X_{K}
$$

and its invariant dual basis $\left\{\chi^{I}\right\}$ which satisfies the Mauer-Cartan equation

$$
d \chi^{I}=\frac{1}{2} C_{J K}^{I} \chi^{J} \wedge \chi^{K}
$$

The metric $g$ on $M$ can be expressed in terms of $\chi^{I}$ as

$$
d s^{2}=g_{I J} \chi^{I} \chi^{J}
$$

where $g_{I J}$ is a nonsingular constant matrix. Thus the Riemannian metric of a 
simply homogeneous manifold can be completely specified by the Lie algebra of $G_{3}$ and a constant matrix $g_{I J}$.

The three-dimensional real Lie algebras are completely classified into the wellknown Bianchi types. They are denoted as I, II, III, IV, V, VI $(A)$, VII $(A)$, VIII and IX. Here VI $(A)$ and VII $(A)$ are one-parameter families of algebras and III is isomorphic to $\mathrm{VI}(A=1)$. They are subdivided into class $\mathrm{A}$ and $\mathrm{B}$ according to whether the trace of the structure constant $a_{I} \equiv \frac{1}{2} C_{I J}^{J}$ has a vanishing norm $\left(\delta^{I J} a_{I} a_{J}\right)^{1 / 2}$ or not. Class A consists of I, II, VI (0), VII (0), VIII and IX, while the other types, IV, V, III $=\mathrm{VI}(1), \mathrm{VI}(A \neq 0)$, VII $(A \neq 0)$ belong to class $\mathrm{B}$.

For a given Bianchi Lie algebras I IX, there exists a unique (up to a constant matrix $\left.g_{I J}\right)$ simply-connected Riemannian manifold $M$ diffeomorphic to a simplyconnected Lie group $G$ which is uniquely determined by the given algebra. $G$ is then a group of isometries acting simply-transitively on $M$. Therefore, for a simply-connected and simply homogeneous manifold with a homogeneity group $G_{3}$, its topology is determined by the Bianchi type of the Lie algebra of $G_{3}$ while its metric is given by (3). Especially, $M$ is diffeomorphic to either $\mathbf{R}^{3}$ or $S^{3}$ depending on whether $G_{3}$ is of the type I VIII or IX respectively.

However, it is not adequate for a general study of spatially homogeneous spacetimes to restrict only on those simply homogeneous manifolds as spatial homogeneous sections. In fact, when one can construct a compact manifold by identifying certain points in a simply homogeneous manifold, it usually lowers the dimension of the group of isometries so that the resulting manifold is not simply homogeneous any longer. For example, a homogeneous spatial section in every class B Bianchi model cannot be simply homogeneous if it is compact, as we shall see later. Therefore we should also include in our consideration a locally homogeneous manifold whose universal covering space is simply homogeneous. More explicitly, we shall consider a wider class of spatially homogeneous space-times as follows. A threedimensional Lie group $G_{3}$ is now assumed to act on the universal covering space of a space-time as a group of isometries, such that each orbit is a spacelike hyper- 
surface $\widetilde{\Sigma}$ on which $G_{3}$ acts simply-transitively. Then $G_{3}$ acts simply-transitively on each $\widetilde{\Sigma}$ as a group of isometries of $\widetilde{\Sigma}$, so the underlying manifold $\Sigma$ is a locally homogeneous manifold with a homogeneity group $G_{3}$. We will concentrate on the study of $\Sigma$ in the following, which is denoted by $(M, g)$. Henceforth we assume that $M$ is closed.

Let us now study in general a locally homogeneous 3-manifold $(M, g)$ with a homogeneity group $G_{3}$. Consider its universal covering space $(\widetilde{M}, \widetilde{g})$ with a covering map $p$. A covering transformation is a homeomorphism $\gamma: \widetilde{M} \rightarrow \widetilde{M}$ such that $p \circ \gamma=p$. The set of covering transformations is a group under composition, which is called a covering transformation group $\Gamma$. Because in this case $\Gamma$ is a discrete subgroup of $\operatorname{Isom}(\widetilde{M}, \widetilde{g})$, acting freely and properly discontinuously on $\widetilde{M}$, $M$ is isometric to the quotient space $\widetilde{M} / \Gamma$. Thus in order to study $M$ one has to examine $\Gamma$ and $\widetilde{M} . \widetilde{M}$ is, as shown above, simply homogeneous so that its structure is determined by the homogeneity group $G_{3}$. Note that $G_{3}$ is in general a subgroup of $\operatorname{Isom}(\widetilde{M}, \widetilde{g})$. In what follows, we will classify all the possible geometries of $(\widetilde{M}, \widetilde{g})$ by employing a modern viewpoint of "geometry" and show some relations between those geometries and the Bianchi types. How one can choose $\Gamma$ to obtain a compact locally homogeneous manifold $M \cong \widetilde{M} / \Gamma$ depends on each class of geometry.

In a modern approach, "geometry" can be viewed in the following way (see [3][4][5], for example). Suppose that $X$ is a manifold and $G$ is a group acting on $X$. $G$ is assumed to act transitively on $X$ with compact point stabilizer (the isotropy subgroup of $G$ at any point of $X$ is compact). Then a "geometry" is the pair $(X, G)$ and the properties of $X$ invariant under the action of $G$. One can recover the ordinary viewpoint of differential geometry by finding a $G$-invariant metric on $X$. (Its existence is guaranteed since $G$ 's stabilizer is compact at every point. And as $G$ acts transitively on $X$, the metric is complete.) In general, there would be many different $G$-invariant metrics on $X$ so that $X$ can have a variety of properties. For instance, as is easily seen from above, the universal covering $(\widetilde{M}, \widetilde{g})$ of a locally homogeneous $(M, g)$ and its isometry group $\operatorname{Isom}(\widetilde{M}, \widetilde{g})$ are an example of $(X, G)$. 
Thurston [4] classified all the three-dimensional geometries under the following restrictions. In this classification, two geometries $(X, G)$ and $\left(X^{\prime}, G^{\prime}\right)$ are defined to be equivalent if there is a diffeomorphism of $X$ with $X^{\prime}$ which casts the action of $G$ on $X$ onto that of $G^{\prime}$ on $X^{\prime}$. First of all, $X$ is assumed simply-connected for one can always study universal covering spaces if necessary. Secondly, we restrict ourselves to the case that $G$ is maximal. It means that when two groups $G_{1}$ and $G_{2}$ such that $G_{1} \subset G_{2}$ can both act on $X$, one should take $G_{2}$ as $G$. In our consideration above, $G=\operatorname{Isom}(\widetilde{M}, \widetilde{g})$ is maximal when one chooses $\widetilde{g}$ appropriately by changing $g_{I J}$ in the metric (3) so that the full isometry group $\operatorname{Isom}(\widetilde{M}, \widetilde{g})$ becomes maximum. So, for example, $\left(E^{3}, \mathbf{R}^{3}\right)$ is out of consideration, where $E^{3}$ is the Euclidean space and $\mathbf{R}^{3}$ acts on it as translations. Rather, one should take the full isometry group of $E^{3}$, namely the three-dimensional Euclidean group $E(3)$, as $G$. Finally, it is assumed that $G$ has a subgroup $\Gamma$ which acts on $X$ as a covering group so that $X / \Gamma$ becomes compact. Then the geometry is said to admit a compact quotient. Now the Thurston's theorem can be stated as follows:

Theorem (Thurston) Any maximal and simply-connected three-dimensional geometry which admits a compact quotient is equivalent to one of the eight geometries $(X, I \operatorname{som} X)$ described below.

(i) $X=S^{3}$, the spherical geometry. $\operatorname{Isom} X=S O(4)$.

(ii) $X=E^{3}$, the Euclidean geometry. $\operatorname{Isom} X=E(3)^{+}$.

(iii) $X=H^{3}$, the hyperbolic geometry. Isom $X=P S L(2, \mathbf{C})$.

(iv) $X=S^{2} \times E^{1}$. $\operatorname{Isom} X=\left(\operatorname{Isom} S^{2} \times \operatorname{Isom} E^{1}\right)^{+}$.

(v) $X=H^{2} \times E^{1}$. $\operatorname{Isom} X=\left(\operatorname{Isom} H^{2} \times \operatorname{Isom} E^{1}\right)^{+}$.

(vi) $X=\widetilde{T_{1}\left(H^{2}\right)}$, the universal covering space of unit tangent space of $H^{2}$. $\operatorname{Isom} X=\operatorname{Isom} H^{2} \times \mathbf{R}$.

(vii) $X=N i l$, the Heisenberg group.

(viii) $X=S o l$, a solvable three-dimensional Lie group. 
Some remarks follow. Here $\operatorname{Isom} X$ means only orientation preserving isometries. $\Gamma$ should be a discrete subgroup of this $\operatorname{Isom} X$ in order that $X / \Gamma$ be orientable. The isometries corresponding to (vii) and (viii) are omitted. The dimension of Isom $X$ is 6 for (i) (iii), 4 for (iv) (vii), and 3 for (viii). Nil and Sol spaces are three-dimensional Lie groups whose Lie algebra is of Bianchi type II and VI $(0)$ respectively. $\widetilde{T_{1}\left(H^{2}\right)}$ is equivalent to $\widetilde{S L_{2} \mathbf{R}}$ which can be also regarded as the universal covering of the Lie group of Bianchi type VIII. Final remark is that all the possibilities of a compact quotients are known for all the classes except the hyperbolic geometry (iii). For complete information, the reader should refer to [4][5].

Returning to our study of locally homogeneous manifolds, we recall that the homogeneity group $G_{3}$ is assumed to be a subgroup of $\operatorname{Isom}(\widetilde{M}, \widetilde{g})$ for any metric of the form (3). Since in the Thurston's classification $G=\operatorname{Isom}(\widetilde{M}, \widetilde{g})$ must be maximal, $G_{3}$ is a subgroup of $G$ which belongs to one of the eight classes. From this we can deduce two interesting consequences.

(A) No anisotropic expansion is allowed for Bianchi model $\mathrm{V}$ with a closed spatial section.

Consider the universal covering space $(\widetilde{M}, \widetilde{g})$ of a closed spatial section that is a locally homogeneous spacelike hypersurface in Bianchi V space-time. Since $(\widetilde{M}, \widetilde{g})$ is simply homogeneous, the metric $\widetilde{g}$ is of the form (3). According to Milnor [10](Special Example 1.7), for the Lie algebra of Bianchi type $\mathrm{V},(\widetilde{M}, \widetilde{g})$ is necessarily isometric to a maximally symmetric space with a negative constant curvature of a certain magnitude, whatever one chooses as $g_{I J}$ in (3). Equivalently, $(\widetilde{M}, \widetilde{g})$ is always the hyperbolic geometry (iii) in the above classification. This fact means that each locally homogeneous spacelike hypersurface in Bianchi V space-time is locally isometric to a negative constant curvature space. But it does not immediately imply that anisotropic expansion is impossible (e.g. it does not for Bianchi I model whose homogeneous spatial section is flat). For there possibly remains a continuous choice of the covering transformation group $\Gamma$ for constructing $M \cong \widetilde{M} / \Gamma$. In 
other words, we may have the freedom of "moduli" of a closed manifold. However, no such freedom arises in this hyperbolic geometry due to the Mostow rigidity theorem (see [3][4]). The theorem asserts that if two closed manifolds with hyperbolic geometry are homeomorphic to each other, they are actually isometric to each other. So a Bianchi $\mathrm{V}$ homogeneous universe is rigid allowing only a change of overall scale factor. Thus the proposition was proved.

(B) Bianchi IV and VI $(A \neq 0,1)$ with a closed spatial section does not exist.

The proof is easy. The homogeneity group must be a subgroup of one of the eight isometry groups in the classification. However, it cannot be for the homogeneity group of Bianchi type IV or VI $(A \neq 0,1)$, as is explicitly shown by examining the Lie algebras of them and the above eight classes. Therefore, no closed Bianchi IV and $\operatorname{VI}(A \neq 0,1)$ exists.

We can proceed further and study the correspondence between the Bianchi models and the Thurston's eight geometries. The result is summarized in Table 1 and Table 2. This can be obtained from the consideration of the proof of the Thurston's classification theorem [5]. From the table it can be seen that a closed locally homogeneous manifold with the homogeneity group VII $(A \neq 0)$ admits the hyperbolic geometry as the case of $\mathrm{V}$ so that no anisotropic expansion is admitted also for this type VII $(A \neq 0)$. Also note that the geometry of (iv) $S^{2} \times E^{1}$ corresponds to the Kantowski-Sachs model, rather than the Bianchi models considered here.

Essentially the same correspondence between the Thurston's classification and the Bianchi types was presented by Fagundes [7]. The fact (B) was also mentioned in it. However, it was assumed that the covering transformation group $\Gamma$ is always a discrete subgroup of the homogeneity group $G_{3}$. (This is not true in general in our assumptions, and causes a substantial difference for class B models as is explained below.) And our approach here is a group theoretical one which clarifies the relation among $G_{3}, \Gamma$ and the eight maximal groups in the Thurston's classification. Such 
an approach is also useful in studying the compactification problem of class B models, as we shall see in the sequel.

On the other hand, in a more general study of Bianchi cosmology with compact spatial sections, Ashtekar and Samuel [8] showed that class B Bianchi universe cannot be closed under a certain condition. We first describe the condition and then put it into a group theoretical expression. By doing it, we can examine the possibility for the compactification of class B models more closely.

On a locally homogeneous manifold $(M, g)$, in general, one can define an invariant basis only locally on each patch of its open coverings $\left\{U_{i}\right\}$. Because each patch $U_{i}$ is isometric to an open neighborhood $\widetilde{U}_{i}$ in $\widetilde{M}$ where an invariant basis can be globally defined, one can induce an invariant basis on each $U_{i}$. Denote by $X_{I}$ and $X_{I}^{\prime}$ such two induced invariant basis on $U_{i}$ and $U_{j}$. Now, at every point in the overlapping region $U_{i} \cap U_{j} \neq \phi$, suppose that these two basis are related with each other as $X_{I}^{\prime}=X_{J} T^{J}{ }_{I}$ where $T^{I}{ }_{J}$ is a constant matrix. In other words, the transition between the two invariant bases is a Lie algebra automorphism. That this condition holds for every overlaps of $\left\{U_{i}\right\}$ is the additional condition mentioned above. As was proved in [8], any class B models cannot be compactified provided that this condition holds.

The above condition of Lie algebra automorphism can be put into a group theoretical expression if one examines the universal covering space $(\widetilde{M}, \widetilde{g})$ and its covering transformation group $\Gamma$. That is, $\Gamma$ is a subgroup of the normalizer of $G_{3}$ in $\operatorname{Isom}(\widetilde{M}, \widetilde{g})$, or equivalently,

$$
\Gamma \subset N\left(G_{3}\right)
$$

where $N\left(G_{3}\right) \equiv\left\{g \in \operatorname{Isom}(\widetilde{M}, \widetilde{g}) \mid g G_{3} g^{-1}=G_{3}\right\}$. This can be easily seen as follows. Let us pay attention to the identification by the action of $\Gamma$ on $\widetilde{M}$. For definiteness, we assume that $G_{3}$ or $\Gamma$ acts on $\widetilde{M}$ as right transformation $R_{h}: x \rightarrow x h$, $\left(x \in \widetilde{M}, h \in G_{3}, \Gamma\right)$. Fix an element $\gamma \in \Gamma$. An invariant basis $X_{I}$ can be globally expanded on $\widetilde{M}$ by choosing a point $y \in \widetilde{M}$ and setting $X_{I x} \equiv\left(R_{g}\right)_{*} X_{I y}$ at every 
point $x \in \widetilde{M}$, where $g$ is a unique element of $G_{3}$ such that $x=y g$. Now take the point $y \in \widetilde{M}$ and the identified point $y \gamma \in \widetilde{M}$, then

$$
\left(R_{\gamma^{-1}}\right)_{*} X_{I y \gamma}=T_{I}^{J} X_{J y}
$$

Here we have just written the induced vector on the left-hand side as a linear combination of the basis $X_{I}$ at $y$. In the next place, take $x$ and $x \gamma$. Because $G_{3}$ acts simply-transitively on $\widetilde{M}$, there exists a unique element $g \in G_{3}$ such that $x \gamma=(y \gamma) g$. Now supposing that $\Gamma \subset N\left(G_{3}\right)$, there exists an element $g^{\prime} \in G_{3}$ such that $\gamma g=g^{\prime} \gamma$. One has then (a) $x \gamma=y g^{\prime} \gamma$ so that $x=y g^{\prime}$ since $\gamma$ is a covering transformation, and (b) $R_{\gamma^{-1}} R_{g}=R_{g \gamma^{-1}}=R_{\gamma^{-1} g^{\prime}}=R_{g^{\prime}} R_{\gamma^{-1}}$. From these (a) and (b), it follows that

$$
\begin{aligned}
\left(R_{\gamma^{-1}}\right)_{*} & X_{I x \gamma}=\left(R_{g^{\prime}}\right)_{*}\left(R_{\gamma^{-1}}\right)_{*} X_{I y \gamma} \\
& =\left(R_{g^{\prime}}\right)_{*} X_{J y} T_{I}^{J} \\
& =X_{J x} T^{J}{ }_{I}
\end{aligned}
$$

which means that the invariant basis at a point and the induced basis from the identified point are related with each other as a Lie algebra automorphism. This holds for every element $\gamma \in \Gamma$, if $\Gamma \subset N\left(G_{3}\right)$.

From this group theoretical viewpoint, we would like to understand why class B models cannot be compactified under this condition. Again consider the universal covering $(\widetilde{M}, \widetilde{g})$. First of all, we note that the next equality holds:

$$
\mathcal{L}_{X_{I}} \Omega=C_{I} \Omega
$$

where $\Omega$ is the volume element $(1 / 3 !) \epsilon_{I J K} \chi^{I} \wedge \chi^{J} \wedge \chi^{K}$ and $C_{I} \equiv C_{I J}^{J}$, the trace of the structure constant. Since $X_{I}$ is globally defined on $\widetilde{M},(7)$ means that $X_{I}$ generates a 1-parameter group of diffeomorphisms of $\widetilde{M}$ onto itself, which changes the volume. Remember that $C_{I}$ is not zero for class B types. Now we show that 
when (4) holds, a vector field which is defined by $V \equiv X_{I} C_{J} g^{I J} \equiv X_{I} C^{I}$ globally exists on $M$ and it satisfies

$$
\mathcal{L}_{V} \Omega=\left(C_{I} C^{I}\right) \Omega
$$

This (8) follows immediately from (7) because (7) locally holds so does its projection onto $M$. The global existence of $V$ on $M$ can be shown as follows. The covering transformation $\gamma \in \Gamma$ generates a diffeomorphism $\phi: \widetilde{M} \rightarrow \widetilde{M}$ as $\phi=R_{\gamma^{-1}}$. From (6),

$$
(\phi)_{*} V=X_{J} T^{J} C^{I}
$$

In general, a diffeomorphism between two manifolds induces an isomorphism between the tensor fields (of the same rank) on each manifolds. By such an isomorphism $\phi^{\#}$ induced from $\phi,(7)$ is mapped into

$$
\mathcal{L}_{\phi^{\#} X_{I}}\left(\phi^{\#} \Omega\right)=C_{I}\left(\phi^{\#} \Omega\right)
$$

By noting that $\phi^{\#} \Omega=\left(\phi^{-1}\right)^{*} \Omega=\Omega$ since $\gamma \in \Gamma \subset \operatorname{Isom}(\widetilde{M}, \widetilde{g})$ and that $\phi^{\#} X_{I}=$ $\phi_{*} X_{I}=X_{J} T_{I}^{J}$ from (6), we obtain

$$
C_{I}=C_{J} T_{I}^{J}
$$

from which one can easily show that (9) reduces to

$$
(\phi)_{*} V=X_{I} C^{I}=V
$$

This means that $V$ is invariant under the covering transformation, so it is globally defined on $M$. Thus, for a globally defined vector field $V$ on $M$, the equation (8) holds. It then follows that there exists a 1-parameter family of transformations 
$f_{t}:(M, g) \rightarrow(M, g)$, generated by $V$, such that $\left(f_{t}\right)^{*} \Omega=\exp \left\{t\left(C_{I} C^{I}\right)\right\} \Omega$. This implies that

$$
\int_{M} \Omega=\int_{M}\left(f_{t}\right)^{*} \Omega=\exp \left\{t\left(C_{I} C^{I}\right)\right\} \int_{M} \Omega,
$$

which contradicts with the finiteness of the volume of closed $M$. This completes the proof of the proposition. The admissible class B type III, V and VII $(A \neq 0)$ can be compactified in such a way that (4) does not hold.

In particular, there is no class B model whose spatially homogeneous sections are closed and simply homogeneous. For there globally exists an invariant basis on a simply homogeneous manifold, but it implies that $\Gamma \subset G_{3}$. This particular case can be shown more easily. A simple calculation shows that the following identity holds for an integral of a spatial divergence,

$$
\int_{M} d^{3} x \partial_{i}\left(|\chi| X_{I}^{i}\right)=C_{I} \int_{M} \Omega,
$$

where $|\chi|$ is a determinant for the matrix $\chi_{i}^{I}$. Since the invariant basis is globally defined in this case, it gives a contradiction for a closed class B model. In fact, such a spatial divergence arises as typical boundary terms in the variation of an action for general Bianchi models. For class B, open or closed, it implies the absence of a general scheme of action principle where one imposes the spatial homogeneity before taking variation of the action, as is well known [9].

Finally, we would like to comment on how such study of closed Bianchi models can be used to investigate global dynamics in $(3+1)$-dimensional gravity.

The canonical formalism of general relativity treats a space-time as a dynamical deformation of a spacelike hypersurface and a time evolution of its threedimensional geometry. It is important to know what are the dynamical degrees of freedom of three-dimensional geometry and how they evolve in time according to the classical equations of motion, in order to understand both classical and quantum aspects of gravity. (2+1)-dimensional gravity serves as a toy model for study- 
ing these things [10][11][12]. In (2+1)-dimension, there are no local gravitationalwave modes but one has global modes, which are related to the so-called "moduli" of a closed 2-manifold. By global dynamics in gravity, we mean such dynamical degrees of freedom that is closely related to the topology of a spatial manifold.

When one proceeds to consider the real life of $(3+1)$-dimensional gravity, it should be first made clear what is global dynamics in $(3+1)$-dimension. It would not be easy to define global deformation of three-dimensional spatial manifold in the full dynamics of $(3+1)$-dimensional gravity. As a first step to understand it, one can restrict one's attention to spatially homogeneous space-times. As we have observed, each spatially homogeneous space is a locally homogeneous manifold which can be recovered from its universal covering space as a quotient space. This is in the same situation as one could define "moduli" of a closed 2-manifold. But since the universal covering space is not always a maximally symmetric space as we saw in Thurston's theorem, it is necessary to distinguish anisotropy from moduli degrees now. Then, one can define moduli of a locally homogeneous 3-manifold by examining the possible quotient spaces of each type of universal covering spaces (this is in progress by another group [13]). It is noted that this viewpoint concentrates only on the geometry of spatial sections so that it may need a certain modification in the space-time construction.

As the next step, one can take into account a deviation from the locally homogeneity presented here. One thing is to include a small fluctuation of local gravitational-wave modes in an appropriate perturbation scheme, or to examine the coupling of the global modes with some matter fields. Another is to consider "topological inhomogeneity". It is conjectured by Thurston [4] that any compact 3-manifold has a kind of prime decomposition, each element of which admits one geometry of the eight classes described. Then it is an interesting problem what is a possible geometry on a manifold that is a connected-sum of such prime manifolds and how one can glue two locally homogeneous geometries of different types along a junction surface in between. 


\section{REFERENCES}

1. M. P. Jr. Ryan and L. C. Shepley, Homogeneous Relativistic Cosmologies, (Princeton University Press, Princeton, 1975).

2. D. Kramer, H. Stephani, M. MacCallum and E. Herlt, Exact Solutions of Einstein's Field Equations, (Cambridge University Press, Cambridge, 1980).

3. W. P. Thurston, The Geometry and Topology of 3-manifolds, to be published by Princeton University Press, 1978/79.

4. W. P. Thurston, Bull. Amer. Math. Soc. 6 (1982) 357.

5. P. Scott, Bull. London Math. Soc. 15 (1983) 401.

6. J. Milnor, Adv. in Math. 21 (1976) 293.

7. H. V. Fagundes, Phys. Rev. Lett. 54 (1985) 1200;

Gen. Rel. Grav. 24 (1992) 199.

8. A. Ashtekar and J. Samuel, Class. Quantum Grav. 8 (1991) 2191.

9. M. A. H. MacCallum and A. H. Taub, Commun. Math. Phys. 25 (1972) 173.

10. V. Moncrief, J. Math. Phys. 30 (1989) 2907.

11. A. Hosoya and K. Nakao, Class. Quantum Grav. 7 (1990) 163;

Prog. Theor. Phys. 84 (1990) 739,

Y. Fujiwara and J. Soda, Prog. Theor. Phys. 83 (1990) 733.

12. T. Okamura and H. Ishihara, Phys. Rev. D46 (1992) 572; to appear in Phys. Rev.

13. A. Hosoya, T. Koike and M. Tanimoto, private communication. 